\title{
Local causality and complete specification: a reply to Seevinck and Uffink
}

\author{
Gábor Hofer-Szabó*
}

\begin{abstract}
A physical theory is called locally causal if any correlation between spacelike separated events is screened-off by local beables completely specifying an appropriately chosen region in the past of the events. In this paper I will define local causality in a clear-cut framework, called local physical theory which integrates both probabilistic and spatiotemporal entities. Then I will argue that, contrary to the claim of Seevinck and Uffink (2011), complete specification does not stand in contradiction to the free variable (no-conspiracy) assumption.
\end{abstract}

Key words: local causality, Bell inequality, complete specification

\section{Introduction}

Local causality is the idea that causal processes propagate though space continuously and with velocity less than the speed of light. John Stewart Bell formulates this intuition in a 1988 interview as follows:

"[Local causality] is the idea that what you do has consequences only nearby, and that any consequences at a distant place will be weaker and will arrive there only after the time permitted by the velocity of light. Locality [= local causality] is the idea that consequences propagate continuously, that they don't leap over distances." (Mann and Crease, 1988)

Bell has returned to this intuitive idea of local causality from time to time and provided a more and more elaborate formulation of it. First he addressed the notion of local causality in his "The theory of local beables" delivered at the Sixth GIFT Seminar in 1975; later in a footnote added to his 1986 paper "EPR correlations and EPW distributions" intending to clean up the first version; and finally in the most elaborate form in his "La nouvelle cuisine" posthumously published in 1990. In this latter paper local causality obtains the following formulation: ${ }^{1}$

"A theory will be said to be locally causal if the probabilities attached to values of local beables in a space-time region $V_{A}$ are unaltered by specification of values of local beables in a spacelike separated region $V_{B}$, when what happens in the backward light cone of $V_{A}$ is already sufficiently specified, for example by a full specification of local beables in a space-time region $V_{C} . "$ (Bell, 1990/2004, p. 239-240)

We reproduce the figure Bell is attaching to his formulation in Fig. 1. (The captation is Bell's original.) Some brief remarks concerning Bell's terminology are in place here (for a detailed analysis see (Norsen 2009, 2011)):

(i) The term "beable" in the quote is Bell's own neologism and is contrasted to the term "observable" used in quantum theory. "The beables of the theory are those entities in it which are, at least tentatively, to be taken seriously, as corresponding to something real" (Bell, 1990/2004, p. 234).

\footnotetext{
* Research Center for the Humanities, Budapest, email: szabo.gabor@btk.mta.hu

${ }^{1}$ For the sake of conformity with the rest of the paper I slightly changed Bell's notation and figure.
} 


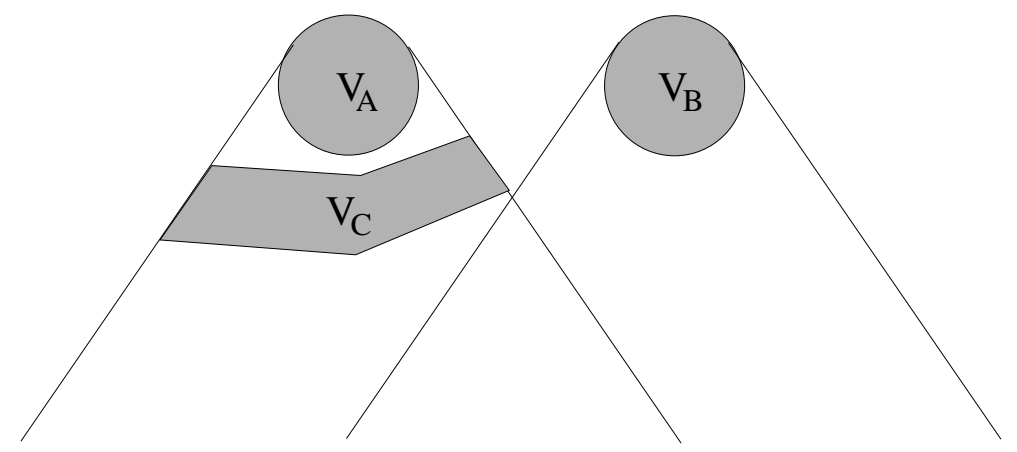

Figure 1: Full specification of what happens in $V_{C}$ makes events in $V_{B}$ irrelevant for predictions about $V_{A}$ in a locally causal theory.

(ii) Beables are to be local: "Local beables are those which are definitely associated with particular space-time regions. The electric and magnetic fields of classical electromagnetism, $\mathbf{E}(t, x)$ and $\mathbf{B}(t, x)$ are again examples." (p. 234).

(iii) Local beables in region $V_{C}$ are to be "fully specified" in order to block causal influences arriving at $V_{A}$ from the common past of $V_{A}$ and $V_{B}$.

This latter point is of central importance and is also stressed by Bell: ${ }^{2}$

"It is important that region $V_{C}$ completely shields off from $V_{A}$ the overlap of the backward light cones of $V_{A}$ and $V_{B}$. And it is important that events in $V_{C}$ be specified completely. Otherwise the traces in region $V_{B}$ of causes of events in $V_{A}$ could well supplement whatever else was being used for calculating probabilities about $V_{A}$. The hypothesis is that any such information about $V_{B}$ becomes redundant when $V_{C}$ is specified completely." (Bell, 1990/2004, p. 240)

In a recent paper Michael Seevinck and Jos Uffink (2011) have questioned the necessary role of complete specification in the definition of local causality and recommended sufficient specification instead. They argue that complete specification is too strong: it contradicts to the so-called no-conspiracy (free variable) condition which requires that the common cause of the correlation be probabilistically independent of the choice of the measurement settings.

I do not see this contradiction and my aim in this paper is to articulate my point. I will proceed as follows. The logical schema of Bell's definition of local causality is the following: if events are localized in the spacetime in such-and-such a way, then these events are to satisfy such-and-such probabilistic independencies. This schema is highly intuitive and easily applicable in the physical praxis, however, in order to account for these inferences from spatiotemporal to probabilistic relations in a mathematically transparent way, one needs to have a framework integrating both spatiotemporal and also probabilistic entities. Only after having such a common framework can one define Bell's notion of local causality in a clear-cut way. Thus, in Section 2 first this framework, called local physical theory, will be introduced and then Bell's notion of local causality will be formulated within this framework. In Section 3 the relation of local causality to the Bell inequalities will be explicated. The main section is Section 4; here it will be argued that there is no tension between complete specification and no-conspiracy. I conclude in Section 5.

\footnotetext{
${ }^{2}$ But, to be fair, see (Bell 1980/2004, p. 106), (Bell 1980/2004, p. 152) and the above (Bell 1990/2004, p. 234) for Bell's hesitation on the issue.
} 


\section{Bell's local causality in a local physical theory}

In developing the notion of a local physical theory one is lead by the following intuitions. A local physical theory is to describe "beables," let them be classical or nonclassical; it is to account for the logical combination of these events; these events should be capable of bearing a probabilistic interpretation; the theory is to provide some way to localize these event in the spacetime, and is also to provide some physically well-motivated principles guiding the association of spacetime regions to physical events; the theory is to guarantee that the symmetries of the spacetime are in tune with the symmetries of the events. (For the details see (Hofer-Szabó and Vecsernyés, 2015 a,b).) All these preliminary intuitions are captured in the following definition (Haag, 1992):

Definition 1. A $\mathcal{P}_{\mathcal{K}}$-covariant local physical theory is a net $\{\mathcal{A}(V), V \in \mathcal{K}\}$ associating algebras of events to spacetime regions which satisfies isotony, microcausality and covariance defined as follows:

1. Isotony. Let $\mathcal{M}$ be a globally hyperbolic spacetime and let $\mathcal{K}$ be a covering collection of bounded, globally hyperbolic subspacetime regions of $\mathcal{M}$ such that $(\mathcal{K}, \subseteq)$ is a directed poset under inclusion $\subseteq$. The net of local observables is given by the isotone map $\mathcal{K} \ni V \mapsto \mathcal{A}(V)$ to unital $C^{*}$-algebras, that is $V_{1} \subseteq V_{2}$ implies that $\mathcal{A}\left(V_{1}\right)$ is a unital $C^{*}$-subalgebra of $\mathcal{A}\left(V_{2}\right)$. The quasilocal algebra $\mathcal{A}$ is defined to be the inductive limit $C^{*}$-algebra of the net $\{\mathcal{A}(V), V \in \mathcal{K}\}$ of local $C^{*}$-algebras.

2. Microcausality (also called as Einstein causality) is the requirement that $\mathcal{A}\left(V^{\prime}\right)^{\prime} \cap \mathcal{A} \supseteq \mathcal{A}(V), V \in \mathcal{K}$, where primes denote spacelike complement and algebra commutant, respectively.

3. Spacetime covariance. Let $\mathcal{P}_{\mathcal{K}}$ be the subgroup of the group $\mathcal{P}$ of geometric symmetries of $\mathcal{M}$ leaving the collection $\mathcal{K}$ invariant. A group homomorphism $\alpha: \mathcal{P}_{\mathcal{K}} \rightarrow$ Aut $\mathcal{A}$ is given such that the automorphisms $\alpha_{g}, g \in \mathcal{P}_{\mathcal{K}}$ of $\mathcal{A}$ act covariantly on the observable net: $\alpha_{g}(\mathcal{A}(V))=\mathcal{A}(g \cdot V), V \in \mathcal{K}$.

If the quasilocal algebra $\mathcal{A}$ of the local physical theory is commutative, we speak about a local classical theory, if it is noncommutative, we speak about a local quantum theory. For local classical theories microcausality fulfills trivially.

A state $\phi$ in a local physical theory is defined as a normalized positive linear functional on the quasilocal observable algebra $\mathcal{A}$. The corresponding GNS representation $\pi_{\phi}: \mathcal{A} \rightarrow \mathcal{B}\left(\mathcal{H}_{\phi}\right)$ converts the net of $C^{*}$ algebras into a net of $C^{*}$-subalgebras of $\mathcal{B}\left(\mathcal{H}_{\phi}\right)$. Closing these subalgebras in the weak topology one arrives at a net of local von Neumann observable algebras: $\mathcal{N}(V):=\pi_{\phi}(\mathcal{A}(V))^{\prime \prime}, V \in \mathcal{K}$. Von Neumann algebras are generated by their projections, which are called quantum events since they can be interpreted as $0-1$-valued observables. The net $\{\mathcal{N}(V), V \in \mathcal{K}\}$ of local von Neumann algebras given above also obeys isotony, microcausality, and $\mathcal{P}_{\mathcal{K}}$-covariance, hence we can also refer to a net $\{\mathcal{N}(V), V \in \mathcal{K}\}$ of local von Neumann algebras as a local physical theory.

Now, a local physical theory is locally causal in Bell's sense if any correlation between spatially separated events is screened off by "local beables" which are localized in a "shielding-off" region and which "completely specify" that region. How to translate Bell's terms of "local beable" and "complete specification" into the language of a local physical theory?

In a classical field theory beables are characterized by sets of field configurations. Taking the equivalence classes of those field configurations which have the same field values on a given spacetime region one can generate local (cylindrical) $\sigma$-algebras. Translating $\sigma$-algebras into the language of abelian von Neumann algebras one can represent Bell's notion of "local beables" in the framework of local physical theories. In a more general way, one can also use the term "local beables" both for abelian and non-abelian local von Neumann algebras, hence treating local classical and quantum theories on an equal footing. Translating "local beables" simply as "elements of a local algebra" naturally brings with it the translation of the term "a complete specification of beables" as "an atomic event of a local algebra" (Henson, 2013). To be sure, here it is assumed that the local algebras of the net are atomic, which is typically not the case, 
for example, in Poincaré covariant algebraic quantum field theory. (For the relation between $\sigma$-algebras and von Neumann algebras and for a more general definition of local causality see (Hofer-Szabó and Vecsernyés, $2015 \mathrm{a}, \mathrm{b}$ ).) With these notions in hand now one can formulate Bell's notion of local causality in a local physical theory as follows:

Definition 2. A local physical theory represented by a net $\{\mathcal{N}(V), V \in \mathcal{K}\}$ of von Neumann algebras is called locally causal (in Bell's sense), if for any pair $A \in \mathcal{N}\left(V_{A}\right)$ and $B \in \mathcal{N}\left(V_{B}\right)$ of projections supported in spacelike separated regions $V_{A}, V_{B} \in \mathcal{K}$ and for every locally normal and faithful state $\phi$ establishing a correlation $\phi(A B) \neq \phi(A) \phi(B)$ between $A$ and $B$, and for any spacetime region $V_{C}$ such that

(i) $V_{C} \subset J_{-}\left(V_{A}\right)$,

(ii) $V_{A} \subset V_{C}^{\prime \prime}$,

(iii) $J_{-}\left(V_{A}\right) \cap J_{-}\left(V_{B}\right) \cap\left(J_{+}\left(V_{C}\right) \backslash V_{C}\right)=\emptyset$,

(see Fig. 2) and for any atomic event $C_{k}$ of $\mathcal{A}\left(V_{C}\right)(k \in K)$, the following holds:

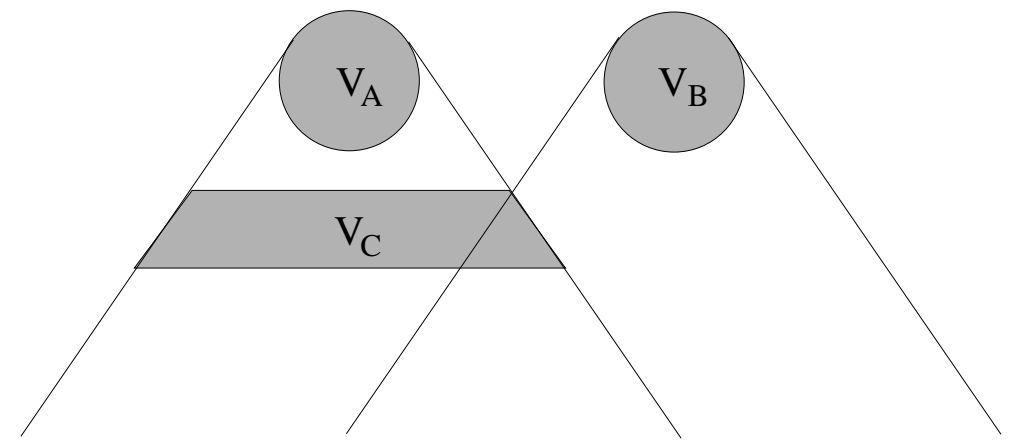

Figure 2: A region $V_{C}$ satisfying Requirements (i)-(iii).

$$
\frac{\phi\left(C_{k} A B C_{k}\right)}{\phi\left(C_{k}\right)}=\frac{\phi\left(C_{k} A C_{k}\right)}{\phi\left(C_{k}\right)} \frac{\phi\left(C_{k} B C_{k}\right)}{\phi\left(C_{k}\right)}
$$

\section{Remarks:}

1. A locally normal state is a normal state on the local von Neumann algebras. A locally faithful state $\phi$ means that any projection $A \in \mathcal{P}(\mathcal{N}(V))$ in the local von Neumann algebra $\mathcal{N}(V)$ has nonzero expectation value. In case of local classical theories a locally faithful state $\phi$ determines uniquely a locally nonzero probability measure $p$ by $p(A):=\phi(A), A \in \mathcal{P}(\mathcal{N}(V))$. By means of this (1) can be written in the following 'symmetric' form:

$$
p\left(A B \mid C_{k}\right)=p\left(A \mid C_{k}\right) p\left(B \mid C_{k}\right)
$$

which further is equivalent to the 'asymmetric' screening-off condition:

$$
p\left(A \mid B C_{k}\right)=p\left(A \mid C_{k}\right)
$$

sometimes used in the literature (for example in (Bell, 1975/2004, p. 54)).

2. The role of Requirement (iii) in the definition is to ensure that " $V_{C}$ shields off from $V_{A}$ the overlap of the backward light cones of $V_{A}$ and $V_{B}$ ". A spacetime region above $V_{C}$ in the common past of the correlating events (see Fig. 3) namely may contain stochastic events which could establish a correlation between $A$ and $B$ in a classical stochastic theory (Norsen, 2011; Seevinck and Uffink 2011). Requirement (iii) is somewhat weaker than Bell's original localization (see Fig. 1) which can be formulated as: 


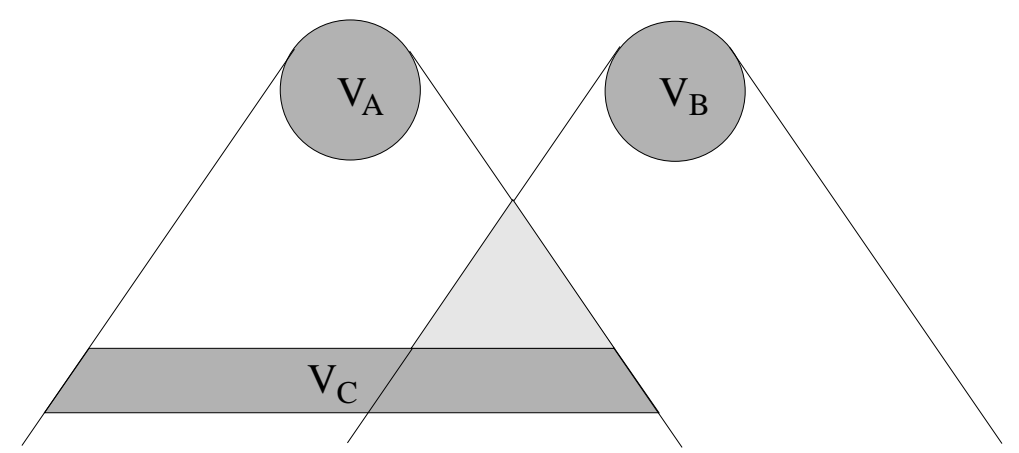

Figure 3: A region $V_{C}$ for which Requirement (iii) does not hold.

(iv) $J_{-}\left(V_{A}\right) \cap J_{-}\left(V_{B}\right) \cap V_{C}=\emptyset$

The difference is that Requirement (iii) does, but Requirement (iv) does not allow for region $V_{C}$ to penetrate into the 'top part' of the common past. However, both requirements coincide, if $V_{C}$ 'shrinks down' to a Cauchy surface. In local classical theories it suffices to use Requirement (iii).

Finally, note that the question whether a given local classical or quantum theory is locally causal is a highly nontrivial question depending on such factors as the atomicity of the local algebras, the fulfilment of the so-called local primitive causality, ${ }^{3}$ or whether there exists a causal dynamics in the theory, etc. (For the details see again (Hofer-Szabó and Vecsernyés, 2015 a,b).)

Next I turn to the relation of Bell's local causality to the Bell inequalities.

\section{Local causality and the Bell inequalities}

From this section on we restrict ourselves to local classical theories since beables are standardly taken to be classical entities. Consider a local classical theory represented by a net $\{\mathcal{N}(V), V \in \mathcal{K}\}$ of local abelian von Neumann algebras. Suppose that Bell's local causality holds in this theory. Let $V_{A}$ and $V_{B}$ be two spatially separated regions in $\mathcal{M}$, and $V_{C}$ a third region (see Fig. 4) such that

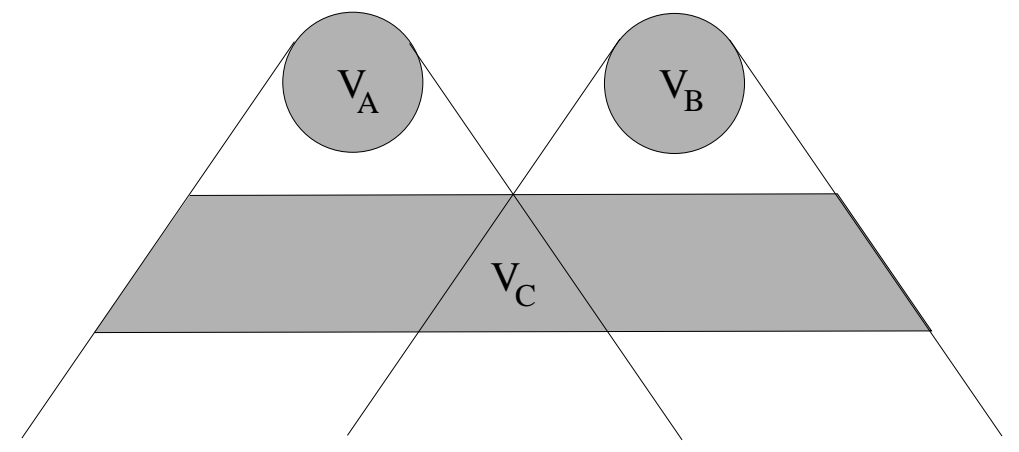

Figure 4: Localization of regions $V_{A}, V_{B}$ and $V_{C}$.

$$
\begin{array}{r}
V_{C} \subset J_{-}\left(V_{A} \cup V_{B}\right) \\
\left(V_{A} \cup V_{B}\right) \subset V_{C}^{\prime \prime} \\
J_{-}\left(V_{A}\right) \cap J_{-}\left(V_{B}\right) \cap\left(J_{+}\left(V_{C}\right) \backslash V_{C}\right)=\emptyset
\end{array}
$$

\footnotetext{
${ }^{3}$ For any globally hyperbolic bounded subspacetime regions $V \in \mathcal{K}, \mathcal{A}\left(V^{\prime \prime}\right)=\mathcal{A}(V)$.
} 
Divide $V_{C}$ into six regions $V_{C}^{L}, V_{C^{\prime}}^{L}, V_{C}^{M}, V_{C^{\prime}}^{M}, V_{C}^{R}$ and $V_{C^{\prime}}^{R}$, for example as depicted in Fig. 5. Here the superscripts $L, M$ and $R$ stand for 'left', 'middle' and 'right', representing those parts of $V_{C}$ which fall into region $J_{-}\left(V_{A}\right) \backslash J_{-}\left(V_{B}\right), J_{-}\left(V_{A}\right) \cap J_{-}\left(V_{B}\right)$ and $J_{-}\left(V_{B}\right) \backslash J_{-}\left(V_{A}\right)$, respectively. Now, let the

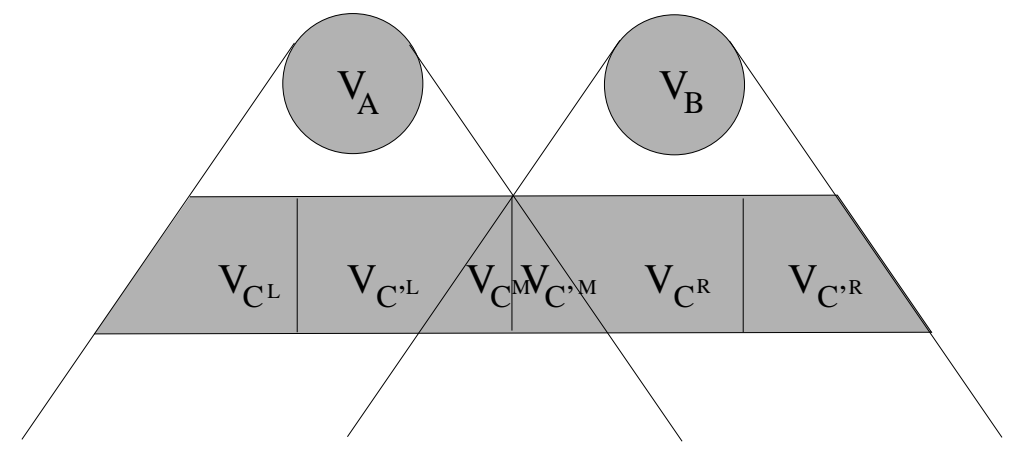

Figure 5: Dividing up region $V_{C}$.

various events be localized in these regions as follows. Let $A_{i}$ and $B_{j}$ be measurement outcomes and $a_{i}$, $b_{j}$ measurement choices localized in the appropriate regions: $A_{i}, a_{i} \in \mathcal{A}\left(V_{A}\right), B_{j}, b_{j} \in \mathcal{A}\left(V_{B}\right)$. (See Fig. 6.) Let, furthermore, $C_{k}^{L}, C_{l}^{L L}, C_{m}^{M}, C_{n}^{\prime M}, C_{p}^{R}, C_{q}^{\prime R}$ be atomic events (minimal projections) in $\mathcal{A}\left(V_{C}^{L}\right)$,

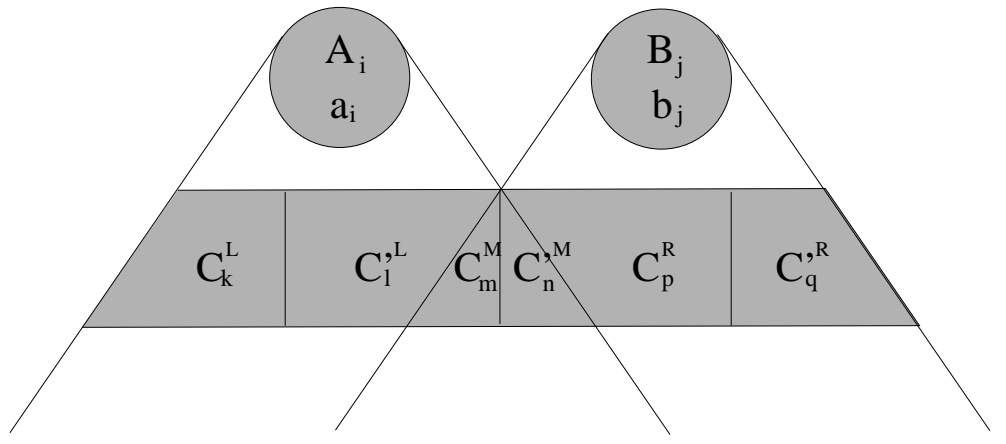

Figure 6: Localization of the various events.

$\mathcal{A}\left(V_{C^{\prime}}^{L}\right), \mathcal{A}\left(V_{C}^{M}\right), \mathcal{A}\left(V_{C^{\prime}}^{M}\right), \mathcal{A}\left(V_{C}^{R}\right)$ and $\mathcal{A}\left(V_{C^{\prime}}^{R}\right)$, respectively, where the indices $i, j, k \ldots$ are taken from appropriate index sets. Now, the difference between the primed and the unprimed events in $V_{C}$ is that the primed events probabilistically depend on the the measurement choices $a_{i}$ and $b_{j}$, whereas the unprimed events are probabilistically completely independent of them:

$$
\begin{aligned}
p\left(a_{i} b_{j} C_{l}^{L} C_{m}^{M} C_{p}^{R}\right) & =p\left(a_{i}\right) p\left(b_{j}\right) p\left(C_{l}^{L}\right) p\left(C_{m}^{M}\right) p\left(C_{p}^{R}\right) \\
p\left(a_{i} b_{j} C_{l}^{L} C_{m}^{M}\right) & =p\left(a_{i}\right) p\left(b_{j}\right) p\left(C_{l}^{L}\right) p\left(C_{m}^{M}\right) \\
& \cdots \\
p\left(a_{i} b_{j} C_{p}^{R}\right) & =p\left(a_{i}\right) p\left(b_{j}\right) p\left(C_{p}^{R}\right)
\end{aligned}
$$

Let us call these conditions no-conspiracy conditions.

To sum up, here we assume that any of the left, middle and right region of $V_{C}$, respectively can be decomposed into two subregions such that each of these subregions contains exclusively either events 'influencing' the measurement choices or events being independent of them. Obviously, only this latter class of events can be regarded as the common cause of the correlation between the measurement outcomes; the former events are playing a role in fixing the measurement settings. As we will see later, this 
assumption of the decomposability of $V_{C}$ into six regions is too tolerant if our aim is to derive the Bell inequalities. It will turn out that there are only five regions, the middle region can contain only unprimed events.

Now, local causality of local physical theory represented by a net $\{\mathcal{N}(V), V \in \mathcal{K}\}$ implies (among others) the following conditional independencies:

$$
\begin{aligned}
p\left(A_{i} a_{i} \mid B_{j} b_{j} C_{k}^{L} C_{l}^{\prime L} C_{m}^{M} C_{n}^{\prime M} C_{p}^{R} C_{q}^{\prime R}\right) & =p\left(A_{i} a_{i} \mid C_{k}^{L} C_{l}^{L} C_{m}^{M} C_{n}^{\prime M}\right) \\
p\left(B_{j} b_{j} \mid C_{k}^{L} C_{l}^{\prime L} C_{m}^{M} C_{n}^{M} C_{p}^{R} C_{q}^{\prime R}\right) & =p\left(B_{j} b_{j} \mid C_{m}^{M} C_{n}^{\prime M} C_{p}^{R} C_{q}^{\prime R}\right) \\
p\left(a_{i} \mid b_{j} C_{k}^{L} C_{l}^{\prime L} C_{m}^{M} C_{n}^{\prime M} C_{p}^{R} C_{q}^{\prime R}\right) & =p\left(a_{i} \mid C_{k}^{L} C_{l}^{\prime L} C_{m}^{M} C_{n}^{\prime M}\right) \\
p\left(b_{j} \mid C_{k}^{L} C_{l}^{\prime L} C_{m}^{M} C_{n}^{\prime M} C_{p}^{R} C_{q}^{\prime R}\right) & =p\left(b_{j} \mid C_{m}^{M} C_{n}^{\prime M} C_{p}^{R} C_{q}^{\prime R}\right)
\end{aligned}
$$

which together with the complete independence of the events $C_{k}^{L}, C_{l}^{\prime L}, C_{m}^{M}, C_{n}^{\prime M}, C_{p}^{R}$ and $C_{q}^{\prime R}$ :

$$
\begin{aligned}
p\left(C_{k}^{L} C_{l}^{L} C_{m}^{M} C_{n}^{\prime M} C_{p}^{R} C_{q}^{\prime R}\right) & =p\left(C_{k}^{L}\right) p\left(C_{l}^{L}\right) p\left(C_{m}^{M}\right) p\left(C_{n}^{\prime M}\right) p\left(C_{p}^{R}\right) p\left(C_{q}^{\prime R}\right) \\
p\left(C_{k}^{L} C_{l}^{L} C_{m}^{M} C_{n}^{\prime M} C_{p}^{R}\right) & =p\left(C_{k}^{L}\right) p\left(C_{l}^{L}\right) p\left(C_{m}^{M}\right) p\left(C_{n}^{\prime M}\right) p\left(C_{p}^{R}\right) \\
& \cdots \\
p\left(C_{p}^{R} C_{q}^{\prime R}\right) & =p\left(C_{p}^{R}\right) p\left(C_{q}^{\prime R}\right)
\end{aligned}
$$

yield the following screening-off or factorization conditions:

$$
\begin{aligned}
p\left(A_{i} B_{j} \mid a_{i} b_{j} C_{k}^{L} C_{l}^{L L} C_{m}^{M} C_{n}^{\prime M} C_{p}^{R} C_{q}^{\prime R}\right) & =p\left(A_{i} \mid a_{i} C_{k}^{L} C_{l}^{L L} C_{m}^{M} C_{n}^{\prime M}\right) p\left(B_{j} \mid b_{j} C_{m}^{M} C_{n}^{\prime M} C_{p}^{R} C_{q}^{\prime R}\right) \\
p\left(A_{i} B_{j} \mid a_{i} b_{j} C_{k}^{L} C_{m}^{M} C_{n}^{\prime M} C_{p}^{R}\right) & =p\left(A_{i} \mid a_{i} C_{k}^{L} C_{m}^{M} C_{n}^{\prime M}\right) p\left(B_{j} \mid b_{j} C_{m}^{M} C_{n}^{\prime M} C_{p}^{R}\right) \\
p\left(A_{i} B_{j} \mid a_{i} b_{j} C_{l}^{L} C_{m}^{M} C_{n}^{\prime M} C_{q}^{\prime R}\right) & =p\left(A_{i} \mid a_{i} C_{l}^{\prime L} C_{m}^{M} C_{n}^{\prime M}\right) p\left(B_{j} \mid b_{j} C_{m}^{M} C_{n}^{\prime M} C_{q}^{\prime R}\right) \\
p\left(A_{i} B_{j} \mid a_{i} b_{j} C_{m}^{M} C_{n}^{\prime M}\right) & =p\left(A_{i} \mid a_{i} C_{m}^{M} C_{n}^{\prime M}\right) p\left(B_{j} \mid b_{j} C_{m}^{M} C_{n}^{\prime M}\right)
\end{aligned}
$$

(For the proof see Appendix A.) These equations show that not only the atomic events $C_{k}^{L} C_{l}^{\prime L} C_{m}^{M} C_{n}^{\prime M} C_{p}^{R} C_{q}^{\prime R}$ localized in the entire $V_{C}$ screen off the conditional correlation

$$
p\left(A_{i} B_{j} \mid a_{i} b_{j}\right) \neq p\left(A_{i} \mid a_{i}\right) p\left(B_{j} \mid b_{j}\right)
$$

but one can freely sum up for any of the primed and unprimed events both in the left and the right region without vitiating the screening-off. In other words, the non-atomic (coarse-grained) events $C_{k}^{L} C_{m}^{M} C_{n}^{\prime M} C_{p}^{R}$, $C_{l}^{\prime L} C_{m}^{M} C_{n}^{\prime M} C_{q}^{\prime R}$ and $C_{m}^{M} C_{n}^{\prime M}$, respectively localized in appropriate subregions of $V_{C}$ will all be screeneroffs for the correlation (23). ${ }^{4}$ That one can freely sum up for both the primed and the unprimed events is a consequence of the fact that in the derivation of (19)-(22) no-conspiracy (7)-(10) does not play a role.

However, for events localized in the middle region one cannot sum up! As a consequence, one cannot get rid of the primed terms $C_{n}^{\prime M}$ in equations (19)-(22). So for example it will not be generally true that

$$
p\left(A_{i} B_{j} \mid a_{i} b_{j} C_{m}^{M}\right)=p\left(A_{i} \mid a_{i} C_{m}^{M}\right) p\left(B_{j} \mid b_{j} C_{m}^{M}\right)
$$

(See Appendix B.) However, if we cannot get rid of the primed terms $C_{n}^{\prime M}$, we will not be able to derive the Bell inequalities since in the derivation we need to use no-conspiracy (7)-(10) which holds only for the unprimed terms. (See Appendix C.)

This shows that our decomposition of region $V_{C}$ into six regions was too liberal. We have to make one step back and restrict our previous schema to the one depicted in Fig. 7. Outside the common past of the correlating events one can have both primed and unprimed events that is events influencing the measurement choices and events being independent of them. However, within the common past there can

\footnotetext{
${ }^{4}$ Note again that the term 'common cause' is used only for those screener-offs which are composed of unprimed events.
} 


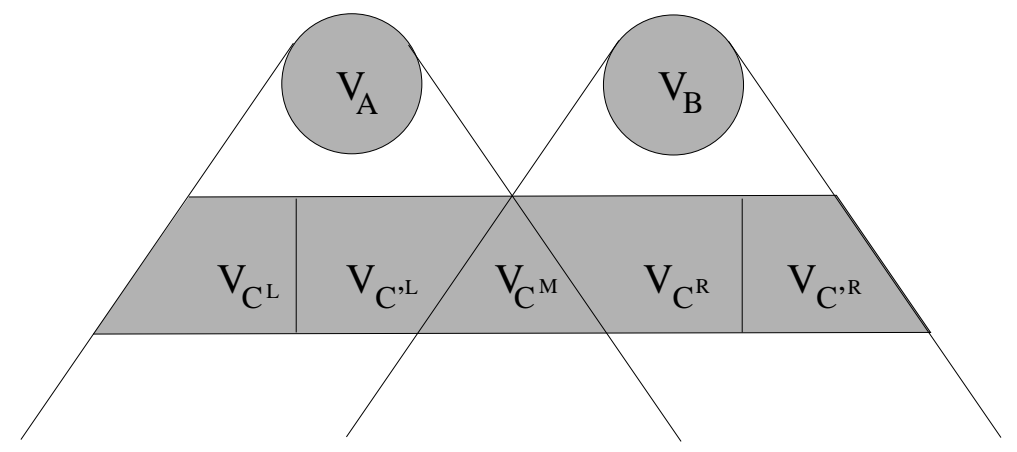

Figure 7: The most general scenario from which the Bell inequalities can be derived.

be only events which are probabilistically independent of the measurement choices. Within this schema the Bell inequalities can be derived.

To sum up, given a locally causal local classical theory represented by a net $\{\mathcal{N}(V), V \in \mathcal{K}\}$ with regions localized as in Fig. 7 and elements in the appropriate regions, complete independence (15)-(18) and no-conspiracy (7)-(10) together imply the Bell inequalities.

\section{Complete versus sufficient specification}

Now I turn to the question of 'complete versus sufficient specification' raised by Norsen (2009) and unfolded by Seevinck and Uffink (2011). In his illuminating paper, comparing the notion of 'completeness' used in Bell's vs. Jarrett's writings, Norsen (2009) raised the following concern: ${ }^{5}$ Since "the past light cones of [the measurement choices] $a$ and $b$ overlap with the region containing $C$ - and $C$ by definition is supposed to contain a complete specification of beables in this region ... one wonders how $a$ and $b$ could possibly not be causally influenced by $C$ (in a locally causal theory)" (Norsen 2009, p. 283.) Seevinck and Uffink take Norsen's point and argue that complete specification is too strong "when formalising the notion of local causality. It is only needed that the specification is sufficiently specified, in the relevant sense" (p. 5); and then they go on to develop this relevant sense in terms of Fisher's statistical concept of sufficiency.

The argument of Seevinck and Uffink against complete specification is put in the form of a dilemma:

" $C$ cannot be expected to be a complete specification of region $V_{C}$ because one must allow for the possibility of traces in region $V_{C}$ of the causal past of both the settings [measurement choices], and given the independence of $C$ and the settings, these traces cannot be included in $C$.

An alternative understanding of this point is that one is here faced with a dilemma. That is, the following two assumptions cannot both hold: (i) the free variables [no-conspiracy] assumption, and (ii) the assumption that $C$ is completely specified, i.e., contains the description of all and every beable in region $V_{C} . "$ (Seevinck and Uffink, 2011, p. 5)

In brief, the complete specification of region $V_{C}$ contradicts to the no-conspiracy condition since if $C$ completely specifies region $V_{C}$, then it also specifies the measurement choices $a$ and $b$, and hence $C$ and $a, b$ cannot be probabilistically independent.

I see, however, no contradiction between complete specification and no-conspiracy. I have a weaker and a stronger claim supporting my point. I start with the weaker one. The upshot of this weaker claim

\footnotetext{
${ }^{5}$ Again for the sake of consistency I changed the notation of both Norsen (2009) and Seevinck and Uffink (2011).
} 
is that the events which satisfy complete specification need not be the same as the events which satisfy no-conspiracy.

Complete specification of a spacetime region, as said before, is simply an atomic event in that region. If our "candidate theory" represented by a net of local algebras is given, then to every bounded region $V_{C}$ there is an algebra $\mathcal{A}\left(V_{C}\right)$ associated; and if the algebra is atomic, the complete specifications that is the atomic events of the region are also given. Consider region $V_{C}$ in Fig. 7. The event $C_{k}^{L} C_{l}^{\prime L} C_{m}^{M} C_{p}^{R} C_{q}^{\prime R}$ is a complete specification in $V_{C}$, but the unprimed event $C_{k} C_{m} C_{p}$ and the primed event $C_{l}^{\prime} C_{q}^{\prime R}$ separately are not. These latter two play different theoretical roles: No-conspiracy holds for $C_{k} C_{m} C_{p}$, therefore it is interpreted as a (possible) common cause of the conditional correlation (23). For $C_{l}^{\prime} C_{q}^{\prime R}$ no-conspiracy does not hold (and a fortiori neither does for the complete specification $C_{k}^{L} C_{l}^{\prime L} C_{m}^{M} C_{p}^{R} C_{q}^{\prime R}$ ). Thus $C_{l}^{\prime} C_{q}^{\prime R}$ has another interpretation: it allows "for the possibility of traces in region $V_{C}$ of the causal past of both the settings." This 'division of labor' between the unprimed $C_{k} C_{m} C_{p}$ and the primed $C_{l}^{\prime} C_{q}^{\prime R}$, however, is no worry: together they provide a complete specification of region $V_{C}$ and enable the derivation of the Bell inequalities as long as the middle region, $V_{C} \cap V_{A} \cap V_{B}$ contains no primed term violating no-conspiracy. In short, in order to derive the Bell inequalities from local causality, those events which completely specify region $V_{C}$ need not be the same events as those satisfying no-conspiracy.

But here is my stronger claim: they can. Namely, there is no contradiction between complete specification and no-conspiracy even if we require them to hold for the same events. To see this, simply consider the case when the subregions $V_{C^{\prime}}^{L}$ and $V_{C^{\prime}}^{R}$ are empty, that is when $V_{C}$ contains exclusively unprimed elements (see Fig. 8). In this case the event $C_{k}^{L} C_{m}^{M} C_{p}^{R}$ will both completely specify region $V_{C}$ and sat-

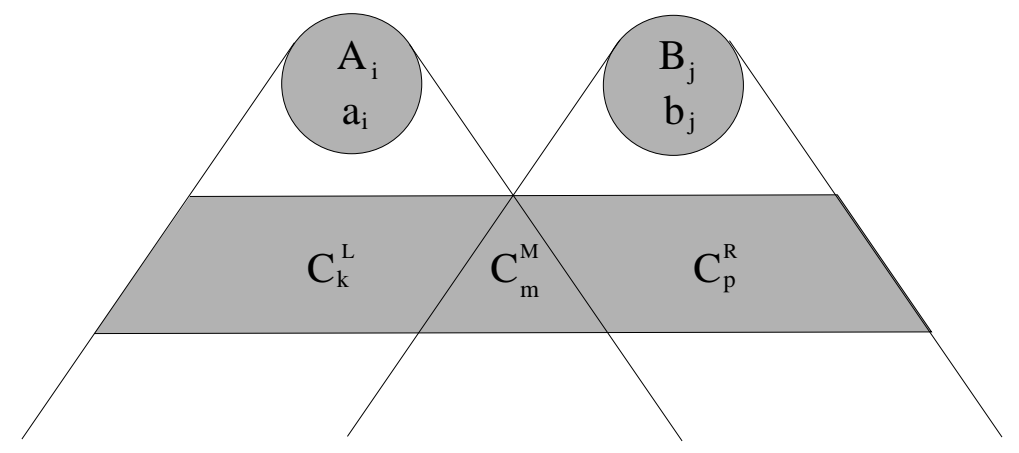

Figure 8: No contradiction between complete specification and no-conspiracy.

isfy no-conspiracy. Consequently, the Bell inequalities will follow. More importantly, this independence between the common causes and the measurement choices does not trivialize the theory, for example by dissolving the conditional correlation (23) between the measurement outcomes.

The next proposition illustrates this latter point.

Proposition 1. There exists a locally causal local classical theory with events $A_{i}, a_{i} \in \mathcal{A}\left(V_{A}\right), B_{j}, b_{j} \in$ $\mathcal{A}\left(V_{B}\right)$ in spatially separated regions $V_{A}$ and $V_{B}$ conditionally correlating in the sense of (23), and atomic events $C_{k}^{L} \in \mathcal{A}\left(V_{C}^{L}\right), C_{m}^{M} \in \mathcal{A}\left(V_{C}^{M}\right)$ and $C_{p}^{R} \in \mathcal{A}\left(V_{C}^{R}\right)$, where $V_{C}=V_{C}^{L} \cup V_{C}^{M} \cup V_{C}^{R}$ satisfies requirements (4)-(6), such that no-conspiracy (7)-(10), moreover complete independence (15)-(18) hold.

Proof. Let $A_{i}, a_{i}, B_{j}, b_{j}, C_{k}^{L}, C_{m}^{M}$ and $C_{p}^{R}$ be events localized as in Fig. 8. Suppose that for the atomic events $C_{k}^{L}, C_{m}^{M}$ and $C_{p}^{R}$ completely specifying region $V_{C}$ both complete independence

$$
p\left(C_{k}^{L} C_{m}^{M} C_{p}^{R}\right)=p\left(C_{k}^{L} C_{m}^{M}\right) p\left(C_{p}^{R}\right)=p\left(C_{k}^{L}\right) p\left(C_{m}^{M} C_{p}^{R}\right)=p\left(C_{m}^{M}\right) p\left(C_{k}^{L} C_{p}^{R}\right)=p\left(C_{k}^{L}\right) p\left(C_{m}^{M}\right) p\left(C_{p}^{R}\right)
$$

and also no-conspiracy

$$
p\left(a_{i} b_{j} C_{k}^{L} C_{m}^{M} C_{p}^{R}\right)=p\left(a_{i} b_{j}\right) p\left(C_{k}^{L} C_{m}^{M} C_{p}^{R}\right)=\cdots=p\left(a_{i}\right) p\left(b_{j}\right) p\left(C_{k}^{L}\right) p\left(C_{m}^{M}\right) p\left(C_{p}^{R}\right)
$$


hold for any combination of the indices. Let the net containing the events be locally causal; for example let

$$
p\left(A_{i} B_{j} \mid a_{i} b_{j} C_{k}^{L} C_{m}^{M} C_{p}^{R}\right)=p\left(A_{i} \mid a_{i} C_{k}^{L} C_{m}^{M}\right) p\left(B_{j} \mid b_{j} C_{m}^{M} C_{p}^{R}\right)=\left(p_{i}^{L} \delta_{1 k} \delta_{1 m}\right)\left(p_{j}^{R} \delta_{1 m} \delta_{1 p}\right)
$$

where $\sum_{i} p_{i}^{L}=\sum_{j} p_{j}^{R}=1$. Now, the conditional probabilities are given as follows:

$$
\begin{aligned}
p\left(A_{i} \mid a_{i}\right) & =\sum_{k, m} p\left(A_{i} \mid a_{i} C_{k}^{L} C_{m}^{M}\right) p\left(C_{k}^{L} C_{m}^{M}\right)=p_{i}^{L} p\left(C_{1}^{L}\right) p\left(C_{1}^{M}\right) \\
p\left(B_{j} \mid b_{j}\right) & =\sum_{m, p} p\left(B_{j} \mid b_{j} C_{m}^{M} C_{p}^{R}\right) p\left(C_{m}^{M} C_{p}^{R}\right)=p_{j}^{R} p\left(C_{1}^{M}\right) p\left(C_{1}^{R}\right) \\
p\left(A_{i} B_{j} \mid a_{i} b_{j}\right) & =\sum_{k, m, p} p\left(A_{i} B_{j} \mid a_{i} b_{j} C_{k}^{L} C_{m}^{M} C_{p}^{R}\right) p\left(C_{k}^{L} C_{m}^{M} C_{p}^{R}\right) \\
& =\sum_{k, m, p} p\left(A_{i} \mid a_{i} C_{k}^{L} C_{m}^{M}\right) p\left(B_{j} \mid b_{j} C_{m}^{M} C_{p}^{R}\right) p\left(C_{k}^{L}\right) p\left(C_{m}^{M}\right) p\left(C_{p}^{R}\right) \\
& =p_{i}^{L} p_{j}^{R} p\left(C_{1}^{L}\right) p\left(C_{1}^{M}\right) p\left(C_{1}^{R}\right)
\end{aligned}
$$

Thus, there is a conditional correlation (23) between $A_{i}$ and $B_{j}$ whenever $p\left(C_{1}^{M}\right) \neq 0$ or 1 .

Consequently, there is no contradiction between complete specification and no-conspiracy even if both are applied to the same events, namely the atomic events of the entire $V_{C}$. The measurement choices can be free of the common causes even if the causal past of the region containing them is completely specified. This independence does not abolish the conditional correlation between the measurement outcomes: atomic events can be probabilistically irrelevant to the measurement choices and at the same time relevant to the measurement outcomes. Moreover, the independence of the measurement choices of the atomic events does not mean that the former are not 'determined' (probabilistically) by the latter. They are: the conditional probabilities $p\left(a_{i} b_{j} \mid C_{k}^{L} C_{m}^{M} C_{p}^{R}\right)$ are set in a local physical theory, even if they are equal to $p\left(a_{i} b_{j}\right)$.

Thus, based on these two claims, I think, there is no need to replace 'complete specification' in Bell's definition of local causality by 'sufficient specification'.

\section{Conclusions}

The main claims of this paper were the following:

(i) The definition of Bell's notion of local causality presupposes a clear-cut framework in which probabilistic and spatiotemporal entities can be related. This goal can be met by introducing the notion of a local physical theory represented by an isotone net of algebras.

(ii) In a local classical theory the measurement outcomes, measurement choices and common cause can be localized in the spacetime such that one can derive the Bell inequalities from local causality, no-conspiracy and independence.

(iii) Contrary to the claim of Seevinck and Uffink, there is no need to weaken the requirement of complete specification in the definition of local causality on the ground that it contradicts to no-conspiracy.

Acknowledgements. This work has been supported by the Hungarian Scientific Research Fund OTKA K-100715. 


\section{References}

J.S. Bell, "Beables for quantum field theory," TH-2053-CERN, presented at the Sixth GIFT Seminar, Jaca, 2-7 June (1975); reprinted in (Bell, 2004, 52-62).

J.S. Bell, "EPR correlations and EPW distributions," in: New Techniques and Ideas in Quantum Measurement Theory, New York Academy of Sciences, (1986); reprinted in (Bell, 2004, 196-200).

J.S. Bell, "La nouvelle cuisine," in: J. Sarlemijn and P. Kroes (eds.), Between Science and Technology, Elsevier, (1990); reprinted in (Bell, 2004, 232-248).

J.S. Bell, Speakable and Unspeakable in Quantum Mechanics, Cambridge: Cambridge University Press, (2004).

R. Haag, Local Quantum Physics, (Springer Verlag, Berlin, 1992).

J. Henson, "Non-separability does not relieve the problem of Bell's theorem," Found. Phys., 43, 1008-1038 (2013).

G. Hofer-Szabó and P. Vecsernyés, "On the concept of Bell's local causality in local classical and quantum theory," (submitted) (2015a).

G. Hofer-Szabó and P. Vecsernyés, "Bell's local causality for philosophers," (submitted) (2015b).

C. Mann and R. Crease, "John Bell, Particle Physicist," (Interview), Omni, 10/8, 84-92 (1988).

T. Norsen, "Local causality and Completeness: Bell vs. Jarrett," Found. Phys., 39, 273 (2009).

T. Norsen, "J.S. Bell's concept of local causality," Am. J. Phys, 79, 12, (2011).

M. P. Seevinck and J. Uffink, "Not throwing out the baby with the bathwater: Bell's condition of local causality mathematically 'sharp and clean', " in: Dieks, D.; Gonzalez, W.J.; Hartmann, S.; Uebel, Th.; Weber, M. (eds.) Explanation, Prediction, and Confirmation The Philosophy of Science in a European Perspective, Volume 2, 425-450 (2011). 


\section{Appendix A}

First we prove equation (22) from local causality (11)-(14) and the complete independence condition (15)-(18):

$$
\begin{aligned}
& p\left(A_{i} B_{j} \mid a_{i} b_{j} C_{m}^{M} C_{n}^{\prime M}\right)=\frac{p\left(A_{i} B_{j} a_{i} b_{j} C_{m}^{M} C_{n}^{\prime M}\right)}{p\left(a_{i} b_{j} C_{m}^{M} C_{n}^{\prime M}\right)} \\
& =\frac{\sum_{k l p q} p\left(A_{i} B_{j} a_{i} b_{j} C_{k}^{L} C_{l}^{L L} C_{m}^{M} C_{n}^{\prime M} C_{p}^{R} C_{q}^{\prime R}\right)}{\sum_{k l p q} p\left(a_{i} b_{j} C_{k}^{L} C_{l}^{\prime L} C_{m}^{M} C_{n}^{\prime M} C_{p}^{R} C_{q}^{\prime R}\right)} \\
& =\frac{\sum_{k l p q} p\left(A_{i} B_{j} a_{i} b_{j} \mid C_{k}^{L} C_{l}^{L} C_{m}^{M} C_{n}^{\prime M} C_{p}^{R} C_{q}^{\prime R}\right) p\left(C_{k}^{L} C_{l}^{\prime L} C_{m}^{M} C_{n}^{\prime M} C_{p}^{R} C_{q}^{\prime R}\right)}{\sum_{k l p q} p\left(a_{i} b_{j} \mid C_{k}^{L} C_{l}^{\prime L} C_{m}^{M} C_{n}^{\prime M} C_{p}^{R} C_{q}^{\prime R}\right) p\left(C_{k}^{L} C_{l}^{\prime L} C_{m}^{M} C_{n}^{\prime M} C_{p}^{R} C_{q}^{\prime R}\right)} \\
& =\frac{\sum_{k l p q} p\left(A_{i} a_{i} \mid B_{j} b_{j} C_{k}^{L} C_{l}^{L} C_{m}^{M} C_{n}^{\prime M} C_{p}^{R} C_{q}^{\prime R}\right) p\left(B_{j} b_{j} \mid C_{k}^{L} C_{l}^{\prime L} C_{m}^{M} C_{n}^{\prime M} C_{p}^{R} C_{q}^{\prime R}\right) p\left(C_{k}^{L} C_{l}^{L} C_{m}^{M} C_{n}^{\prime M} C_{p}^{R} C_{q}^{\prime R}\right)}{\sum_{k l p q} p\left(a_{i} \mid b_{j} C_{k}^{L} C_{l}^{\prime L} C_{m}^{M} C_{n}^{\prime M} C_{p}^{R} C_{q}^{\prime R}\right) p\left(b_{j} \mid C_{k}^{L} C_{l}^{\prime L} C_{m}^{M} C_{n}^{\prime M} C_{p}^{R} C_{q}^{\prime R}\right) p\left(C_{k}^{L} C_{l}^{\prime L} C_{m}^{M} C_{n}^{\prime M} C_{p}^{R} C_{q}^{\prime R}\right)} \\
& \stackrel{(11)-(14)}{=} \frac{\sum_{k l p q} p\left(A_{i} a_{i} \mid C_{k}^{L} C_{l}^{\prime L} C_{m}^{M} C_{n}^{\prime M}\right) p\left(B_{j} b_{j} \mid C_{m}^{M} C_{n}^{\prime M} C_{p}^{R} C_{q}^{\prime R}\right) p\left(C_{k}^{L} C_{l}^{\prime L} C_{m}^{M} C_{n}^{\prime M} C_{p}^{R} C_{q}^{\prime R}\right)}{\sum_{k l p q} p\left(a_{i} \mid C_{k}^{L} C_{l}^{\prime L} C_{m}^{M} C_{n}^{\prime M}\right) p\left(b_{j} \mid C_{m}^{M} C_{n}^{\prime M} C_{p}^{R} C_{q}^{\prime R}\right) p\left(C_{k}^{L} C_{l}^{\prime L} C_{m}^{M} C_{n}^{\prime M} C_{p}^{R} C_{q}^{\prime R}\right)} \\
& \stackrel{(15) \_(18)}{=} \frac{\sum_{k l p q} p\left(A_{i} a_{i} \mid C_{k}^{L} C_{l}^{L L} C_{m}^{M} C_{n}^{\prime M}\right) p\left(B_{j} b_{j} \mid C_{m}^{M} C_{n}^{\prime M} C_{p}^{R} C_{q}^{\prime R}\right) p\left(C_{k}^{L} C_{l}^{L L} C_{m}^{M} C_{n}^{\prime M}\right) p\left(C_{p}^{R} C_{q}^{\prime R}\right)}{\sum_{k l p q} p\left(a_{i} \mid C_{k}^{L} C_{l}^{\prime L} C_{m}^{M} C_{n}^{\prime M}\right) p\left(b_{j} \mid C_{m}^{M} C_{n}^{\prime M} C_{p}^{R} C_{q}^{\prime R}\right) p\left(C_{k}^{L} C_{l}^{\prime L} C_{m}^{M} C_{n}^{\prime M}\right) p\left(C_{p}^{R} C_{q}^{\prime R}\right)} \\
& =\quad\left(\frac{\sum_{k l} p\left(A_{i} a_{i} \mid C_{k}^{L} C_{l}^{L L} C_{m}^{M} C_{n}^{\prime M}\right) p\left(C_{k}^{L} C_{l}^{L L} C_{m}^{M} C_{n}^{\prime M}\right)}{\sum_{k l} p\left(a_{i} \mid C_{k}^{L} C_{l}^{\prime L} C_{m}^{M} C_{n}^{\prime M}\right) p\left(C_{k}^{L} C_{l}^{\prime L} C_{m}^{M} C_{n}^{\prime M}\right)}\right)\left(\frac{\sum_{p q} p\left(B_{j} b_{j} \mid C_{m}^{M} C_{n}^{\prime M} C_{p}^{R} C_{q}^{\prime R}\right) p\left(C_{p}^{R} C_{q}^{\prime R}\right)}{\sum_{p q} p\left(b_{j} \mid C_{m}^{M} C_{n}^{\prime M} C_{p}^{R} C_{q}^{\prime R} C_{p}^{R}\right) p\left(C_{p}^{R} C_{q}^{\prime R}\right)}\right) \\
& =\quad\left(\frac{\sum_{k l} p\left(A_{i} a_{i} \mid C_{k}^{L} C_{l}^{L L} C_{m}^{M} C_{n}^{\prime M}\right) p\left(C_{k}^{L} C_{l}^{L L} C_{m}^{M} C_{n}^{\prime M}\right)}{\sum_{k l} p\left(a_{i} \mid C_{k}^{L} C_{l}^{\prime L} C_{m}^{M} C_{n}^{\prime M}\right) p\left(C_{k}^{L} C_{l}^{\prime L} C_{m}^{M} C_{n}^{\prime M}\right)}\right)\left(\frac{\sum_{p q} p\left(B_{j} b_{j} \mid C_{m}^{M} C_{n}^{\prime M} C_{p}^{R} C_{q}^{\prime R}\right) p\left(C_{p}^{R} C_{q}^{\prime R}\right)}{\sum_{p q} p\left(b_{j} \mid C_{m}^{M} C_{n}^{\prime M} C_{p}^{R} C_{q}^{\prime R}\right) p\left(C_{p}^{R} C_{q}^{\prime R}\right)}\right)\left(\frac{p\left(C_{m}^{M} C_{n}^{\prime M}\right)}{p\left(C_{m}^{M} C_{n}^{\prime M}\right)}\right) \\
& \stackrel{(15)-(18)}{=}\left(\frac{\sum_{k l} p\left(A_{i} a_{i} \mid C_{k}^{L} C_{l}^{\prime L} C_{m}^{M} C_{n}^{\prime M}\right) p\left(C_{k}^{L} C_{l}^{\prime L} C_{m}^{M} C_{n}^{\prime M}\right)}{\sum_{k l} p\left(a_{i} \mid C_{k}^{L} C_{l}^{\prime L} C_{m}^{M} C_{n}^{\prime M}\right) p\left(C_{k}^{L} C_{l}^{\prime L} C_{m}^{M} C_{n}^{\prime M}\right)}\right)\left(\frac{\sum_{p q} p\left(B_{j} b_{j} \mid C_{m}^{M} C_{n}^{\prime M} C_{p}^{R} C_{q}^{\prime R}\right) p\left(C_{m}^{M} C_{n}^{\prime M} C_{p}^{R} C_{q}^{\prime R}\right)}{\sum_{p q} p\left(b_{j} \mid C_{m}^{M} C_{n}^{\prime M} C_{p}^{R} C_{q}^{\prime R}\right) p\left(C_{m}^{M} C_{n}^{\prime M} C_{p}^{R} C_{q}^{\prime R}\right)}\right) \\
& =\left(\frac{\sum_{k l} p\left(A_{i} a_{i} C_{k}^{L} C_{l}^{L L} C_{m}^{M} C_{n}^{\prime M}\right)}{\sum_{k l} p\left(a_{i} C_{k}^{L} C_{l}^{\prime L} C_{m}^{M} C_{n}^{\prime M}\right)}\right)\left(\frac{\sum_{p q} p\left(B_{j} b_{j} C_{m}^{M} C_{n}^{\prime M} C_{p}^{R} C_{q}^{\prime R}\right)}{\sum_{p q} p\left(b_{j} C_{m}^{M} C_{n}^{\prime M} C_{p}^{R} C_{q}^{\prime R}\right)}\right) \\
& =\quad\left(\frac{p\left(A_{i} a_{i} C_{m}^{M} C_{n}^{\prime M}\right)}{p\left(a_{i} C_{m}^{M} C_{n}^{\prime M}\right)}\right)\left(\frac{p\left(B_{j} b_{j} C_{m}^{M} C_{n}^{\prime M}\right)}{p\left(b_{j} C_{m}^{M} C_{n}^{\prime M}\right)}\right)=p\left(A_{i} \mid a_{i} C_{m}^{M} C_{n}^{\prime M}\right) p\left(B_{j} \mid b_{j} C_{m}^{M} C_{n}^{\prime M}\right)
\end{aligned}
$$

where the numbers over the equation signs refer to the equation used at that step.

The proof of (21), (20) and (19), respectively can be obtained from the above proof by simply omitting certain summations. For (21) just omit summation for $l$ and $r$; for (20) omit summation for $k$ and $q$; and for (19) omit all four. 


\section{Appendix B}

Here we prove that (24) does not generally hold. The proof follows that in Appendix A, except that here there is an extra summation also for $n$, which causes the trouble in the row below starting with a $\neq$ sign:

$$
\begin{aligned}
& p\left(A_{i} B_{j} \mid a_{i} b_{j} C_{m}^{M}\right)=\frac{p\left(A_{i} B_{j} a_{i} b_{j} C_{m}^{M}\right)}{p\left(a_{i} b_{j} C_{m}^{M}\right)} \\
& =\frac{\sum_{k \ln p q} p\left(A_{i} B_{j} a_{i} b_{j} C_{k}^{L} C_{l}^{L L} C_{m}^{M} C_{n}^{\prime M} C_{p}^{R} C_{q}^{\prime R}\right)}{\sum_{k l n p q} p\left(a_{i} b_{j} C_{k}^{L} C_{l}^{\prime L} C_{m}^{M} C_{n}^{\prime M} C_{p}^{R} C_{q}^{\prime R}\right)} \\
& =\frac{\sum_{k \ln p q} p\left(A_{i} B_{j} a_{i} b_{j} \mid C_{k}^{L} C_{l}^{L L} C_{m}^{M} C_{n}^{\prime M} C_{p}^{R} C_{q}^{\prime R}\right) p\left(C_{k}^{L} C_{l}^{L} C_{m}^{M} C_{n}^{\prime M} C_{p}^{R} C_{q}^{\prime R}\right)}{\sum_{k l n p q} p\left(a_{i} b_{j} \mid C_{k}^{L} C_{l}^{\prime L} C_{m}^{M} C_{n}^{\prime M} C_{p}^{R} C_{q}^{\prime R}\right) p\left(C_{k}^{L} C_{l}^{\prime L} C_{m}^{M} C_{n}^{\prime M} C_{p}^{R} C_{q}^{\prime R}\right)} \\
& =\frac{\sum_{k \ln p q} p\left(A_{i} a_{i} \mid B_{j} b_{j} C_{k}^{L} C_{l}^{L} C_{m}^{M} C_{n}^{\prime M} C_{p}^{R} C_{q}^{\prime R}\right) p\left(B_{j} b_{j} \mid C_{k}^{L} C_{l}^{L L} C_{m}^{M} C_{n}^{\prime M} C_{p}^{R} C_{q}^{\prime R}\right) p\left(C_{k}^{L} C_{l}^{L} C_{m}^{M} C_{n}^{\prime M} C_{p}^{R} C_{q}^{\prime R}\right)}{\sum_{k \ln p q} p\left(a_{i} \mid b_{j} C_{k}^{L} C_{l}^{\prime L} C_{m}^{M} C_{n}^{\prime M} C_{p}^{R} C_{q}^{\prime R}\right) p\left(b_{j} \mid C_{k}^{L} C_{l}^{L L} C_{m}^{M} C_{n}^{\prime M} C_{p}^{R} C_{q}^{\prime R}\right) p\left(C_{k}^{L} C_{l}^{\prime L} C_{m}^{M} C_{n}^{\prime M} C_{p}^{R} C_{q}^{\prime R}\right)} \\
& \stackrel{(11)=(14)}{=} \frac{\sum_{k \ln p q} p\left(A_{i} a_{i} \mid C_{k}^{L} C_{l}^{L L} C_{m}^{M} C_{n}^{\prime M}\right) p\left(B_{j} b_{j} \mid C_{m}^{M} C_{n}^{M} C_{p}^{R} C_{q}^{\prime R}\right) p\left(C_{k}^{L} C_{l}^{\prime L} C_{m}^{M} C_{n}^{\prime M} C_{p}^{R} C_{q}^{\prime R}\right)}{\sum_{k \ln p q} p\left(a_{i} \mid C_{k}^{L} C_{l}^{\prime L} C_{m}^{M} C_{n}^{\prime M}\right) p\left(b_{j} \mid C_{m}^{M} C_{n}^{\prime M} C_{p}^{R} C_{q}^{\prime R}\right) p\left(C_{k}^{L} C_{l}^{\prime L} C_{m}^{M} C_{n}^{\prime M} C_{p}^{R} C_{q}^{\prime R}\right)} \\
& \stackrel{(15) \underset{\ldots}{=}(18)}{=} \frac{\sum_{k \ln p q} p\left(A_{i} a_{i} \mid C_{k}^{L} C_{l}^{L L} C_{m}^{M} C_{n}^{\prime M}\right) p\left(B_{j} b_{j} \mid C_{m}^{M} C_{n}^{\prime M} C_{p}^{R} C_{q}^{\prime R}\right) p\left(C_{k}^{L} C_{l}^{L} C_{m}^{M} C_{n}^{\prime M}\right) p\left(C_{p}^{R} C_{q}^{\prime R}\right)}{\sum_{k \ln p q} p\left(a_{i} \mid C_{k}^{L} C_{l}^{\prime L} C_{m}^{M} C_{n}^{\prime M}\right) p\left(b_{j} \mid C_{m}^{M} C_{n}^{\prime M} C_{p}^{R} C_{q}^{\prime R}\right) p\left(C_{k}^{L} C_{l}^{\prime L} C_{m}^{M} C_{n}^{\prime M}\right) p\left(C_{p}^{R} C_{q}^{\prime R}\right)} \\
& =\frac{\sum_{n}\left(\sum_{k l} p\left(A_{i} a_{i} \mid C_{k}^{L} C_{l}^{L} C_{m}^{M} C_{n}^{\prime M}\right) p\left(C_{k}^{L} C_{l}^{L} C_{m}^{M} C_{n}^{M}\right) \sum_{p q} p\left(B_{j} b_{j} \mid C_{m}^{M} C_{n}^{\prime M} C_{p}^{R} C_{q}^{\prime R}\right) p\left(C_{p}^{R} C_{q}^{\prime R}\right)\right)}{\sum_{n}\left(\sum_{k l} p\left(a_{i} \mid C_{k}^{L} C_{l}^{L L} C_{m}^{M} C_{n}^{\prime M}\right) p\left(C_{k}^{L} C_{l}^{L} C_{m}^{M} C_{n}^{\prime M}\right) \sum_{p q} p\left(b_{j} \mid C_{m}^{M} C_{n}^{\prime M} C_{p}^{R} C_{q}^{\prime R}\right) p\left(C_{p}^{R} C_{q}^{\prime R}\right)\right)} \\
& =\frac{\sum_{n}\left(\sum_{k l} p\left(A_{i} a_{i} \mid C_{k}^{L} C_{l}^{L L} C_{m}^{M} C_{n}^{M}\right) p\left(C_{k}^{L} C_{l}^{L} C_{m}^{M} C_{n}^{\prime M}\right) \sum_{p q} p\left(B_{j} b_{j} \mid C_{m}^{M} C_{n}^{\prime M} C_{p}^{R} C_{q}^{R}\right) p\left(C_{p}^{R} C_{q}^{R}\right)\right)}{\sum_{n}\left(\sum_{k l} p\left(a_{i} \mid C_{k}^{L} C_{l}^{\prime L} C_{m}^{M} C_{n}^{M}\right) p\left(C_{k}^{L} C_{l}^{L} C_{m}^{M} C_{n}^{\prime M}\right) \sum_{p q} p\left(b_{j} \mid C_{m}^{M} C_{n}^{\prime M} C_{p}^{R} C_{q}^{\prime R}\right) p\left(C_{p}^{R} C_{q}^{\prime R}\right)\right)}\left(\frac{p\left(C_{m}^{M} C_{n}^{\prime M}\right)}{p\left(C_{m}^{M} C_{n}^{\prime M}\right)}\right) \\
& \neq \quad\left(\frac{\sum_{k l n} p\left(A_{i} a_{i} \mid C_{k}^{L} C_{l}^{L} C_{m}^{M} C_{n}^{M}\right) p\left(C_{k}^{L} C_{l}^{L L} C_{m}^{M} C_{n}^{\prime M}\right)}{\sum_{k l n} p\left(a_{i} \mid C_{k}^{L} C_{l}^{\prime L} C_{m}^{M} C_{n}^{\prime M}\right) p\left(C_{k}^{L} C_{l}^{L} C_{m}^{M} C_{n}^{\prime M}\right)}\right)\left(\frac{\sum_{n p q} p\left(B_{j} b_{j} \mid C_{m}^{M} C_{n}^{\prime M} C_{p}^{R} C_{q}^{\prime R}\right) p\left(C_{m}^{M} C_{n}^{\prime M}\right) p\left(C_{p}^{R} C_{q}^{\prime R}\right)}{\sum_{n p q} p\left(b_{j} \mid C_{m}^{M} C_{n}^{\prime M} C_{p}^{R} C_{q}^{\prime R}\right) p\left(C_{m}^{M} C_{n}^{\prime M}\right) p\left(C_{p}^{R} C_{q}^{\prime R}\right)}\right)
\end{aligned}
$$

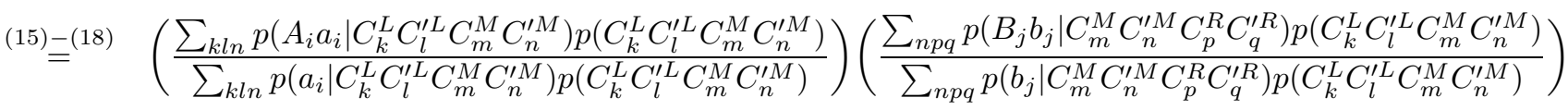

$$
\begin{aligned}
& =\left(\frac{\sum_{k l n} p\left(A_{i} a_{i} C_{k}^{L} C_{l}^{L} C_{m}^{M} C_{n}^{\prime M}\right)}{\sum_{k l n} p\left(a_{i} C_{k}^{L} C_{l}^{\prime L} C_{m}^{M} C_{n}^{\prime M}\right)}\right)\left(\frac{\sum_{n p q} p\left(B_{j} b_{j} C_{m}^{M} C_{n}^{\prime M} C_{p}^{R} C_{q}^{R}\right)}{\sum_{n p q} p\left(b_{j} C_{m}^{M} C_{n}^{\prime M} C_{p}^{R} C_{q}^{\prime R}\right)}\right) \\
& =\left(\frac{p\left(A_{i} a_{i} C_{m}^{M}\right)}{p\left(a_{i} C_{m}^{M}\right)}\right)\left(\frac{p\left(B_{j} b_{j} C_{m}^{M}\right)}{p\left(b_{j} C_{m}^{M}\right)}\right)=p\left(A_{i} \mid a_{i} C_{m}^{M}\right) p\left(B_{j} \mid b_{j} C_{m}^{M}\right)
\end{aligned}
$$

where again the numbers over the equation signs refer to the equation used at that step.

\section{Appendix $\mathrm{C}$}

Here we prove why in the derivation of the Clauser-Horne inequality

$$
-1 \leqslant p\left(A_{i} B_{j} \mid a_{i} b_{j}\right)+p\left(A_{i} B_{j^{\prime}} \mid a_{i} b_{j^{\prime}}\right)+p\left(A_{i^{\prime}} B_{j} \mid a_{i^{\prime}} b_{j}\right)-p\left(A_{i^{\prime}} B_{j^{\prime}} \mid a_{i^{\prime}} b_{j^{\prime}}\right)-p\left(A_{i} \mid a_{i} b_{j}\right)-p\left(B_{j} \mid a_{i} b_{j}\right) \leqslant 0
$$

one should use (24) instead of (22). The standard derivation goes as follows:

It is a simple arithmetic fact that for any $\alpha, \alpha^{\prime}, \beta, \beta^{\prime} \in[0,1]$ :

$$
-1 \leqslant \alpha \beta+\alpha \beta^{\prime}+\alpha^{\prime} \beta-\alpha^{\prime} \beta^{\prime}-\alpha-\beta \leqslant 0
$$


Now let $\alpha, \alpha^{\prime}, \beta, \beta^{\prime}$ first be the conditional probabilities taken from (22):

$$
\begin{aligned}
\alpha & \equiv p\left(A_{i} \mid a_{i} C_{m}^{M} C_{n}^{M}\right) \\
\alpha^{\prime} & \equiv p\left(A_{i^{\prime}} \mid a_{i^{\prime}} C_{m}^{M} C_{n}^{\prime M}\right) \\
\beta & \equiv p\left(B_{j} \mid b_{j} C_{m}^{M} C_{n}^{M}\right) \\
\beta^{\prime} & \equiv p\left(B_{j^{\prime}} \mid b_{j^{\prime}} C_{m}^{M} C_{n}^{\prime M}\right)
\end{aligned}
$$

Plugging (35)-(38) into (34) one obtains

$$
\begin{array}{r}
-1 \leqslant p\left(A_{i} \mid a_{i} C_{m}^{M} C_{n}^{\prime M}\right) p\left(B_{j} \mid b_{j} C_{m}^{M} C_{n}^{\prime M}\right)+p\left(A_{i} \mid a_{i} C_{m}^{M} C_{n}^{\prime M}\right) p\left(B_{j^{\prime}} \mid b_{j^{\prime}} C_{m}^{M} C_{n}^{\prime M}\right) \\
+p\left(A_{i^{\prime}} \mid a_{i^{\prime}} C_{m}^{M} C_{n}^{\prime M}\right) p\left(B_{j} \mid b_{j} C_{m}^{M} C_{n}^{\prime M}\right)-p\left(A_{i^{\prime}} \mid a_{i^{\prime}} C_{m}^{M} C_{n}^{\prime M}\right) p\left(B_{j^{\prime}} \mid b_{j^{\prime}} C_{m}^{M} C_{n}^{\prime M}\right) \\
-p\left(A_{i} \mid a_{i} C_{m}^{M} C_{n}^{\prime M}\right)-p\left(B_{j} \mid b_{j} C_{m}^{M} C_{n}^{\prime M}\right) \leqslant 0
\end{array}
$$

which using (22) transforms into

$$
\begin{array}{r}
-1 \leqslant p\left(A_{i} B_{j} \mid a_{i} b_{j} C_{m}^{M} C_{n}^{\prime M}\right)+p\left(A_{i} B_{j^{\prime}} \mid a_{i} b_{j^{\prime}} C_{m}^{M} C_{n}^{M}\right) \\
+p\left(A_{i^{\prime}} B_{j} \mid a_{i^{\prime}} b_{j} C_{m}^{M} C_{n}^{\prime M}\right)-p\left(A_{i^{\prime}} B_{j^{\prime}} \mid a_{i^{\prime}} b_{j^{\prime}} C_{m}^{M} C_{n}^{\prime M}\right) \\
-p\left(A_{i} \mid a_{i} C_{m}^{M} C_{n}^{\prime M}\right)-p\left(B_{j} \mid b_{j} C_{m}^{M} C_{n}^{\prime M}\right) \leqslant 0
\end{array}
$$

Finally, multiplying the above inequality by $p\left(C_{m}^{M} C_{n}^{M M}\right)$ and summing up for the indices $m, n$ one obtains

$$
\begin{array}{r}
-1 \leqslant \sum_{m n}\left[p\left(A_{i} B_{j} \mid a_{i} b_{j} C_{m}^{M} C_{n}^{\prime M}\right)+p\left(A_{i} B_{j^{\prime}} \mid a_{i} b_{j^{\prime}} C_{m}^{M} C_{n}^{\prime M}\right)\right. \\
+p\left(A_{i^{\prime}} B_{j} \mid a_{i^{\prime}} b_{j} C_{m}^{M} C_{n}^{M}\right)-p\left(A_{i^{\prime}} B_{j^{\prime}} \mid a_{i^{\prime}} b_{j^{\prime}} C_{m}^{M} C_{n}^{M}\right) \\
\left.-p\left(A_{i} \mid a_{i} C_{m}^{M} C_{n}^{\prime M}\right)-p\left(B_{j} \mid b_{j} C_{m}^{M} C_{n}^{\prime M}\right)\right] p\left(C_{m}^{M} C_{n}^{\prime M}\right) \leqslant 0
\end{array}
$$

which is equivalent to (33) only if

$$
p\left(a_{i} b_{j} C_{m}^{M} C_{n}^{\prime M}\right)=p\left(a_{i} b_{j}\right) p\left(C_{m}^{M} C_{n}^{\prime M}\right)
$$

were the case, which is not, since $C_{n}^{\prime M}$ is not independent of $a_{i}$ and $b_{j}$.

Now, starting the whole reasoning again with conditional probabilities taken from (24):

$$
\begin{aligned}
\alpha & \equiv p\left(A_{i} \mid a_{i} C_{m}^{M}\right) \\
\alpha^{\prime} & \equiv p\left(A_{i^{\prime}} \mid a_{i^{\prime}} C_{m}^{M}\right) \\
\beta & \equiv p\left(B_{j} \mid b_{j} C_{m}^{M}\right) \\
\beta^{\prime} & \equiv p\left(B_{j^{\prime}} \mid b_{j^{\prime}} C_{m}^{M}\right)
\end{aligned}
$$

the derivation goes through since instead of (42) one is to use

$$
p\left(a_{i} b_{j} C_{m}^{M}\right)=p\left(a_{i} b_{j}\right) p\left(C_{m}^{M}\right)
$$

which is one of the no-conspiracy conditions (7)-(10). Thus one can use (24) in the derivation of the Clauser-Horne inequality but not (22). 